\title{
Integrated Sensing System for Stamping Monitoring Control
}

\author{
Numpon Mahayotsanun, Jian Cao, Michael Peshkin \\ Department of Mechanical Engineering \\ Northwestern University \\ Evanston, Illinois, USA \\ numorm@northwestern.edu, jcao@northwestern.edu
}

\author{
Sripati Sah, Robert Gao \\ Department of Mechanical and Industrial Engineering \\ University of Massachusetts \\ Amherst, Massachusetts, USA \\ sash@ecs.umass.edu, gao@ecs.umass.edu
}

\begin{abstract}
This paper investigates the feasibility of integrating a draw-in sensor and an array of force sensors under sheet metal tooling surface for the on-line monitoring of stamping operations. The draw-in sensor was developed based on the mutual inductance principle and provided linear results between the draw-in of the tested sheet metal and the induced voltages. The draw-in sensor was tested to simulate the stamping environment. To validate the experimental results, the draw-in sensor tests were compared to both the analytical and the simulation methods and the results were in agreement. Currently, the draw-in sensor is being implemented in a hydraulic stamping press to observe the functionality and accuracy of the draw-in sensor. This paper identifies a new approach to process monitoring in stamping processes by embedding force sensors into the stamping tooling structure. It is aimed to detect stamping process defects through identifying patterns of forming pressure variations. Towards this end experiments were conducted on a stamping test-bed equipped with an array of force sensors. Measurements from the spatially distributed sensors were used to numerically interpolate the pressure distribution at any point on the tooling surface by Thin Plates Splines. The combined accuracy of the physical sensing system and the surface generation technique has been estimated by comparing press force calculated from numerical integration of the TPS determined pressure surfaces with direct measurements.
\end{abstract}

\section{INTRODUCTION}

The physical setup of a stamping operation (Figure 1) consists of three main components: the die, the binder, and the punch. In a stamping operation the punch moves down pressing the workpiece into the die causing plastic deformation of the workpiece material. During the operation the perimeter of the workpiece is confined between the

\author{
C.T. Wang \\ Global Die Center \\ General Motors \\ Michigan, USA \\ chuan-tao.wang@gm.com
}

binder and die flange. The binder force regulates the flow of the workpiece into the die cavity during the process. The planar movement of the workpiece periphery is known as drawn-in. The draw-in amount is an important manufacturing index in sheet metal forming and is an indicator of the success of a forming process [8]. Insufficient draw-in causes splits and excessive thinning in stamped parts while excessive draw-in induces wrinkles and surface defects on the parts. In order to continuously and reliably measure the draw-in amount of sheet metals in a stamping process without damaging the tool, the draw-in sensor was developed.
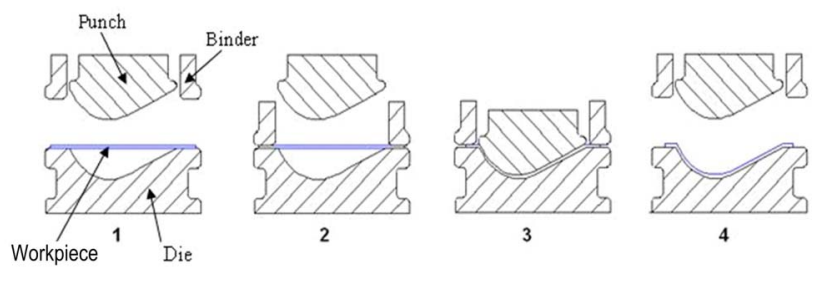

Figure 1. A typical stamping setup

It is known that variations in a stamping operation such as die imbalance, wrinkling, thinning or punch over/under travel lead to local variations in the pressure distribution over the work piece [1]. A direct approach for interpreting the dynamic pressure distribution on the workpiece-tooling interface is through a time indexed series of three dimensional surfaces, each surface representing the forming pressure distribution at a time instant. This method requires the generation of three dimensional surfaces numerically interpolated from discrete sensor measurements. In the present work Thin Plate Splines (TPS) are used for 
estimating the forming pressure distributions from force sensors embedded in a stamping test-bed.

The remainder of this paper is organized into two sections. The first section reports on the development of the draw-in sensor. The evaluation of Thin Plate Splines for interpolating the forming pressure distribution on a sheet metal working surface from a limited number of spatially distributed sensors is presented in the second section.

\section{DRAW-IN SENSING}

\section{A. Experimental setup}

A draw-in sensor was developed using the mutual inductance principle $[1,2]$. The sensor consists of two main components; two transducers and a data acquisition board. A simulated stamping environment was constructed as shown in Figure 2. One of the transducers is inserted in the blankholder slot and the other transducer is located in the bottom die slot. A sheet metal is placed in between and fully covering the two transducers. During the experiment the sheet metal is pulled away from the transducers. The amount of the covering area of the sheet metal results in the change of the induced voltages detected by the transducers. The induced voltage signals are amplified by the data acquisition board and then mapped with the pulling distance of the sheet (representing a draw-in amount.)

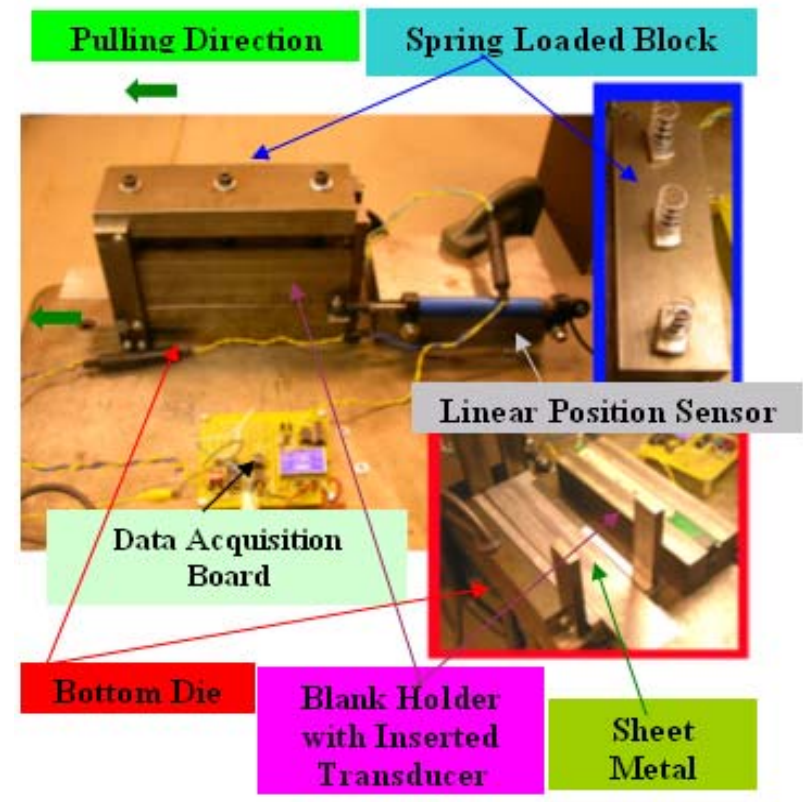

Figure 2. Experimental setup simulating a stamping environment

\section{B. Draw-in sensor results}

The draw-in sensor provides linear results between the induced voltage and the sheet metal displacement as seen in Figure 3 .

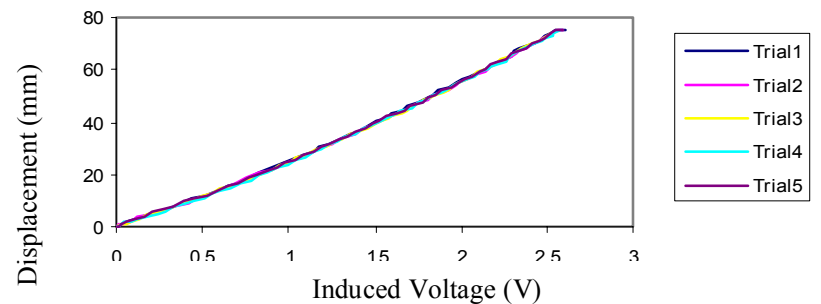

Figure 3. Repeatability results testing a $1.66 \mathrm{~mm}$ thick aluminum 5052 sheet having a $3.32 \mathrm{~mm}$ gap between the $12.7 \mathrm{~mm}$ wide transducers

In addition to the experimental results, the analytical and simulation models of the draw-in sensor were constructed to verify the validity of the experimental results. The comparison among the three methods is shown in Figure 4. The results from all three methods possess similar trend when there is no gap between the sheet metal and the transducers.

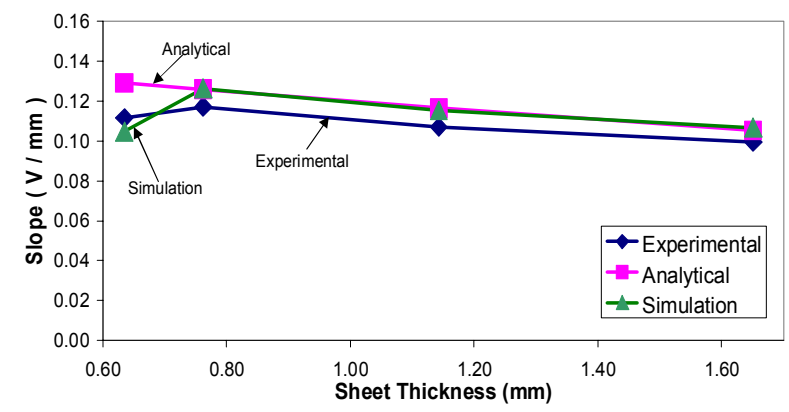

Figure 4. Slope comparison among the experimental, analytical, and simulation results of testing the $12.7 \mathrm{~mm}$ wide transducers with no gap in between

\section{Implementation of the draw-in sensor}

The draw-in sensor will be installed in the hydraulic press at Northwestern University to determine its functionality and accuracy of measuring draw-in amounts. Figure 5 shows the locations of the transducers in the die and binder of the hydraulic press.

Two transducer slots (one in the binder on top of the other in the die)

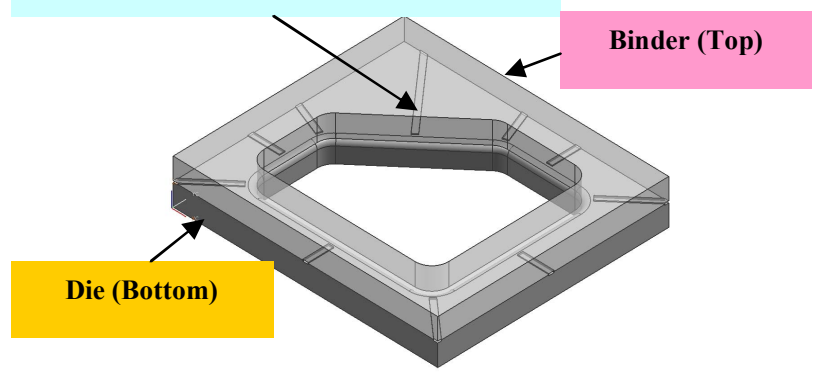

Figure 5. Transducer locations in the die and binder of the hydraulic press 


\section{EMBEDDED FORCE SENSING}

\section{A. Thin Plate Spline Surfaces}

A Thin Plate Spline (TPS) surface is mathematically defined as the unique function $\mathrm{z}=\mathrm{P}(\mathrm{x}, \mathrm{y})$, which minimizes the function:

$$
R(P)=\iint_{R^{2}}\left(P_{x x}^{2}+2 P_{x y}^{2}+P_{y y}^{2}\right) d x d y
$$

The solution of which is of the following form [4, 5]:

$$
z=P(x, y)=\sum_{j=1}^{n} a_{j} E(x, y)+b_{0}+b_{1} x+b_{2} y
$$

Here $a_{j}, b_{0}, b_{1}$, and $b_{2}$ are constants which define the surface and are determined by applying interpolation conditions on Eqn. (2). Symbol ' $n$ ' is the total number of data points, and the function $\mathrm{E}(\mathrm{x}, \mathrm{y})$ is defined as:

$$
\left.\begin{array}{c}
E(x, y)=J\left(\left\|\left(x-x_{j}, y-y_{j}\right)\right\|\right) \\
J(v)=v^{2} \log \left(v^{2}\right) \\
\|\cdot\|=\text { length of vector }
\end{array}\right\}
$$

\section{B. Test Bed Design and TPS Pressure Surfaces}

The test setup consists of two 6" 8 " mating plates of AL6061 each 1.5" thick with an average surface roughness of $22 \mu$ in. The die has a 3" 5 " through slot cut in the middle to aid in sensor placement. The die has been embedded with a rectangular array of eight sensors. The setup is mounted on an Instron hydraulic press installed with a calibrated loaded cell rated for 10,000 lbf. The test setup, sensor placement, and an example of acquired pressure signals are shown in Figure 6. The sensors were sampled at $100 \mathrm{~Hz}$ using a Labview program, the press force was simultaneously recorded from the load cell at the same rate. The test setup was subjected to sinusoidal and delta shaped press loads of different amplitudes.

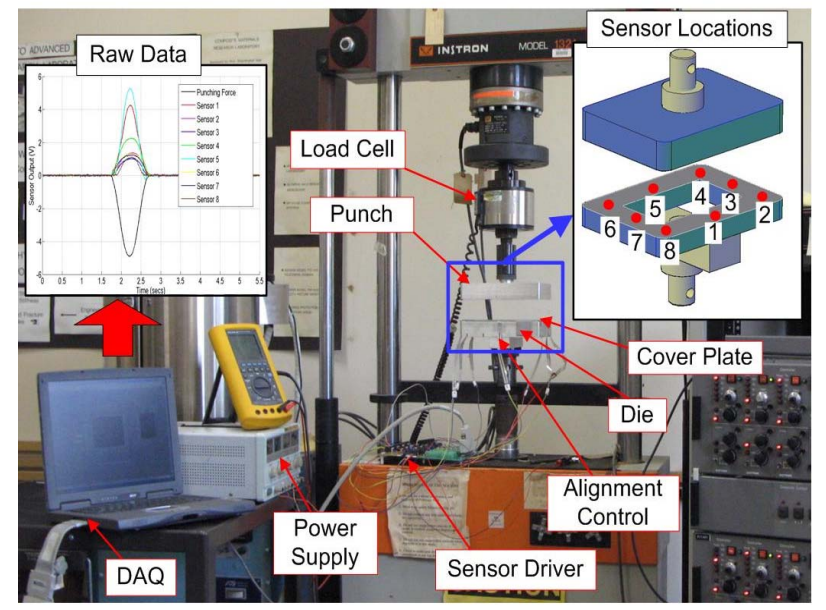

Figure 6. Experimental setup

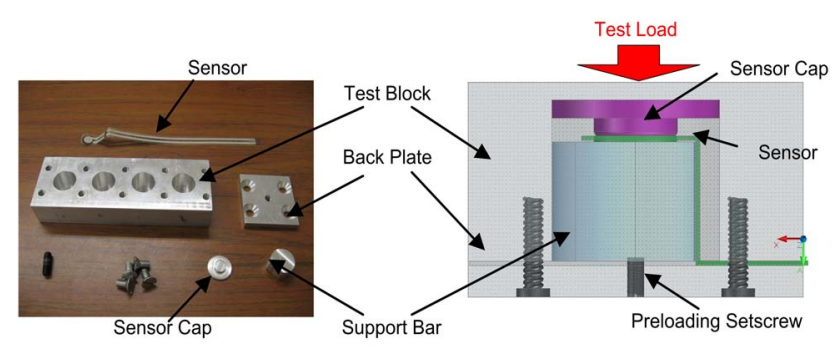

Figure 7. Sensor installation

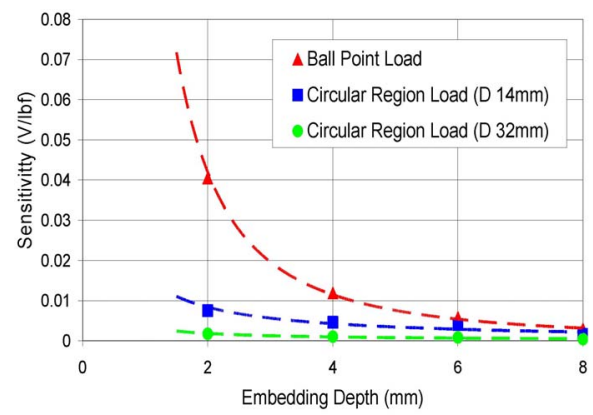

Figure 8. Effect of embedding depth on sensitivity

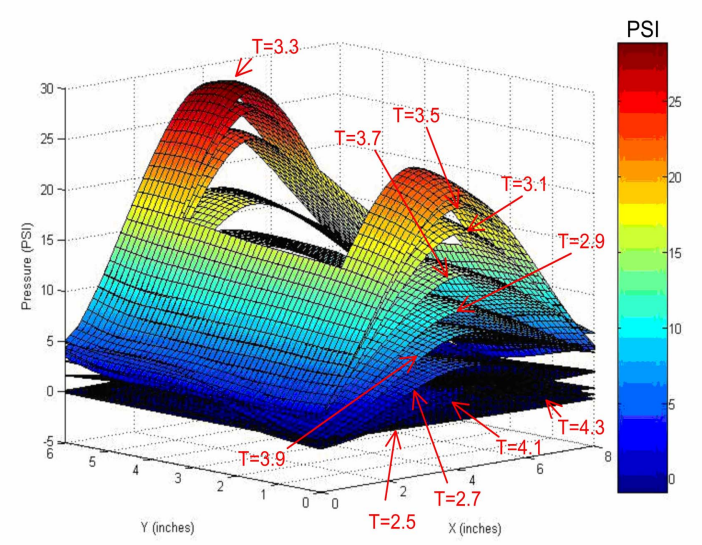

Figure 9. TPS estimated pressure distribution

\section{Accuracy of TPS Estimation}

To evaluate the accuracy of TPS scheme the estimated pressure was numerically integrated over the die surface to determine the net force acting on the working interface. The net force should ideally be equal to the load cell measurement. The difference between the load cell measurement and the surface calculated press force is an indicator of the accuracy of the sensing system. The following expression was used to evaluate the net force:

$$
\mathrm{F}(\mathrm{t})=\sum_{i=1}^{n} \sum_{j=1}^{m} P_{i, j}(t) d A(i, j)
$$


Here $F(t)$ is the estimated press force at time $t$. The constants $\mathrm{n}$ and $\mathrm{m}$ represent the number of divisions into which the die surface is partitioned for numeric integration. The term $\mathrm{dA}(\mathrm{i}, \mathrm{j})$ is the area of the surface element at location $(i, j)$ on the die surface, and $\mathrm{Pi}, \mathrm{j}(\mathrm{t})$ is the pressure acting on the same area element at time $t$. It is noted that Eqn. 4 is valid for planar surfaces. For the calculation of press force from experimental measurements, $\mathrm{n}$ and $\mathrm{m}$ were taken to be 50 .

Figure 10 shows the surface integrated press force and the load cell measured press force for the $0.5 \mathrm{~Hz}$ sinusoidal load. The press force was calculated by integration of TPS pressure surfaces at $100 \mathrm{~ms}$ intervals. Figure 11 shows the same information for a $0.5 \mathrm{~Hz}$ delta load. The estimated and measured values of the press force for the sinusoidal loading are determined to be in $88 \%$ agreement when compared at 30 time instants. In the case of delta loading the agreement is calculated to be $85 \%$. In both the cases difference between estimated and measured press force values is largest when the press force is maximum. In addition the surface calculated press force lags the load cell measured press force during increasing load. However, no lag is observed during load removal. This is effect is attributed to hysteresis in sensor measurements.

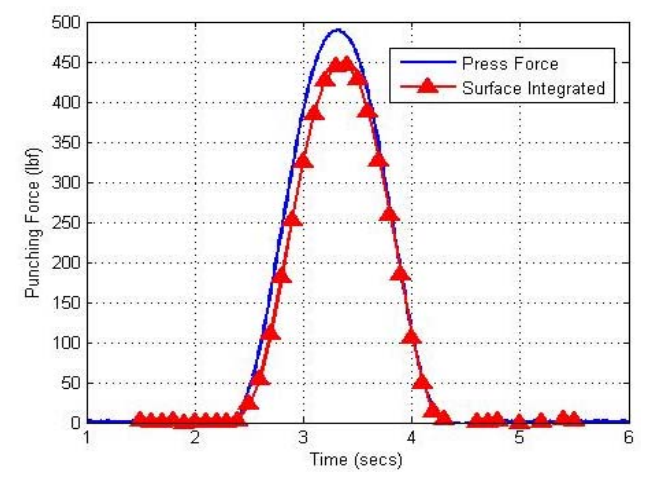

Figure 10. Measured \& estimated press force - Sinusoid load

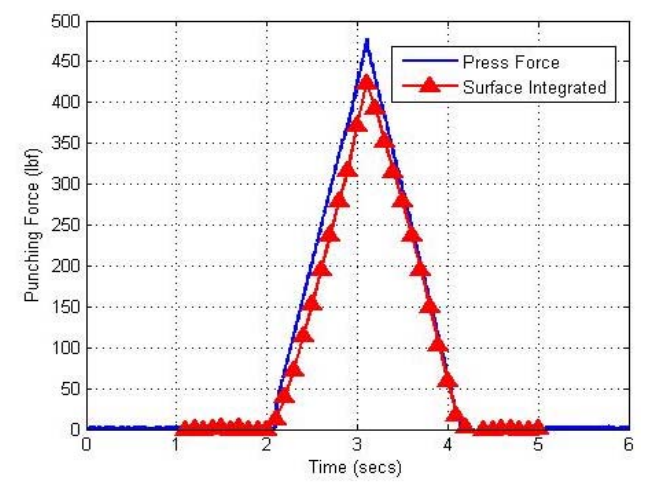

Figure 11. Measured \& estimated press force - Delta load

\section{CONCLUSION}

A draw-in sensor and an embedded pressure sensing scheme have been developed for process monitoring applications in sheet metal stamping operations. The drawin sensor provides linear results between the displacement of the sheet metal and the induced voltages. The analytical and simulation results of the draw-in sensor agree with its experimental results. The draw-in sensor will be implemented in the hydraulic stamping press to determine its functionality. In addition an embedded force sensing technique has been developed for defect detection in the stamping process. The combined accuracy of the physical sensing system and TPS interpolation scheme in estimating pressure distributions in experimental scenarios has been evaluated. It has been determined that changes in the nature of loading do not adversely affect the accuracy of pressure surface estimation. Research is being continued to determine the effects of various types of process defects on the pressure distribution at the workpiece-tooling interface.

\section{ACKNOWLEDGMENT}

The authors gratefully acknowledge funding provided by the National Science Foundation under DMI-0620957 and DMI-0620972.

\section{REFERENCES}

[1] M.C. Doolan, S. Kalyanasundaram, P. Hodgson, and M.J. CardewHall, "Identifying variation in sheet metal," Journal of Materials Processing Technology, vol. 115, pp. 142-146, 2001.

[2] P. Kopczynski, "LVDTs. Theory \& Application," Sensors, vol. 9, pp. 18-22, 1992.

[3] D-J. Choi, C-T Rim, et al., "High Sensitivity Inductive Sensing System for Position Measurement," IEEE Instrumentation and Measurement Technology Conference, vol. 2, pp. 595-599, 2000.

[4] J. Hanchi, M. Li, and B.E. Klamecki, "Sensing of interface loads in manufacturing processes," Tribological Aspects in Manufacturing, American Society of Mechanical Engineers, vol. 2, pp. 203-216, 1991

[5] H. Du, and B.E. Klamecki, "Force sensors embedded in surfaces for manufacturing and other tribological process monitoring," Tribological Aspects in Manufacturing, ASME Journal of Manufacturing Science and Engineering, vol. 121, pp. 739-748, 1999.

[6] G. Wahba, "Spline models for observational data," Captial City Press, 1992.

[7] F. L. Bookstein, "Principal warps: Thin Plate Splines and the decomposition of deformations," IEEE on Pattern Analysis and Machine Intelligence, vol. 11, pp. 567-585, 1989

[8] C.T. Wang, N. Goan, and J. Zhang, "Draw-in Map- a road map for simulation-guided die tryout and stamping process control," The $6^{\text {th }}$ International Conference on 3D Simulations for Sheet Forming Process (NumiSheet'2005) Detroit, USA, pp. 66-69, August 15-19, 2005 